\title{
$\angle S$ Research Square \\ Elderly patients with medial meniscus tear should be aware of the occurrence of osteonecrosis: A case report and literature review
}

\section{Weiming Yang ( $\nabla$ ywmcz@163.com )}

The Second Affiliated Hospital of Guangzhou University of Chinese Medicine

\section{Caiqiong Zhao}

The Second Affiliated Hospital of Guangzhou University of Chinese Medicine

\section{Xuewei Cao}

The Second Affilicated Hospital of Guangzhou University of Chinese Medicine

\section{Research article}

Keywords: Spontaneous osteonecrosis of the knee, Meniscal injury, Misdiagnosis, Unicompartmental knee arthroplasty

Posted Date: January 2nd, 2020

DOI: https://doi.org/10.21203/rs.2.19915/v1

License: (c) (i) This work is licensed under a Creative Commons Attribution 4.0 International License. Read Full License 


\section{Abstract}

Background: Spontaneous osteonecrosis of the knee (SONK) was defined as a distinct clinical entity with characteristical findings that subchondral lesions in the weight-bearing region of a single condyle. In the early stage of SONK, we usually think that injury of meniscus was to blame for the knee pain. Elderly patients with medial meniscus tear should be aware of the occurrence of osteonecrosis.

Case presentation $₫ A$ 67-year-old male complained of pain in the left knee for 3 months in 2013. Magnetic resonance imaging (MRI) revealed a medial meniscal root tear (level III), so that he patient underwent arthroscopic meniscectomy. However, no obvious pain relief achieved at the follow-up. The pain was associated with weight bearing, of which was initially intermittent, gradually progressed in intensity and became frequent even at rest. At 18 months after the first visit, we re-examined the knee MRI and results clearly revealed subchondral bone osteonecrosis of the condyle. Finally, we performed unicompartmental arthroplasty which significantly relieved the pain and restored the walking ability of the patient.

Conclusions: Meniscus injury often coexist with SONK. In the early stages of SONK, the area of necrosis is small which is very easy to be ignored causing a misdiagnosis. The case reminds us should pay much attention to the possibility of subchondral bone necrosis in patients with knee pain and meniscal injury.

\section{Background}

Spontaneous osteonecrosis of the knee (SONK) is defined as a distinct clinical entity with characteristic of subchondral lesion in the weight bearing region of a single condyle, which was first reported by Ahlback in 1968[1, 2]. SONK is prevalent in middle-aged women ( $>55$ years old and male/ female ratio $\approx$ 1:3). Its main symptom is acute pain in the involved knee and often confined to the medial knee. The presence of a medial meniscal tear has been proposed as a potential etiology of SONK. In the early stages of SONK, the area of necrosis is too small to be noticed, which may mislead us to attribute knee pain to meniscus injury so that leading to a misdiagnosis as meniscus injury rather than spontaneous knee osteonecrosis. However, SONK is a slow progressive disease and the illness can gradually worsen if it haven't been properly diagnosed and treated in a timely manner. Here, we report a case of spontaneous knee osteonecrosis which was misdiagnosed as a meniscal injury. The treatment of arthroscopic meniscectomy was invalid while unicompartmental knee arthroplasty successfully relieved the pain and restored the joint function.

\section{Case Presentation}

A 67-year-old male who complained of the left knee pain for about 3 months with no history of trauma was admitted to our institution in december 2013. The pain was confined to the medial condyle, associating with local tenderness, and which was particularly severe on weight bearing. Physical examination demonstrated the joint had no swollen, deformed or instability and the skin temperature was normal, McMurray (+). MRI revealed a well-defined medial meniscal root tear (level III) but no obvious 
subchondral bone lesions in femoral condyle. (Fig. 1). Therefore, the patient underwent a successful arthroscopic meniscectomy. Intraoperatively, the medial meniscal root exhibited a radial tear and all of the lateral meniscus, the articular cartilage and the anterior/ posterior cruciate ligaments were normal. (Fig. 2).

Inexplicably, the patient had no pain relief at the 2 weeks follow-up and painkillers were continuously required. In addition, situation was also the same case at the next 6 months follow-up. Initially, the pain was associated with weight bearing but which gradually got worsen and even appeared at rest. In May 2015 (18 months after the first visiting), X-ray and MRI of the knee were performed again to detect the radiographic changes due to the severe knee pain. The plain $\mathrm{X}$-ray revealed that a radiolucent lesion with a surrounding sclerotic halo combined with subtle flattening were involved in femoral condyle. (Fig. 3). MRI clearly revealed osteonecrosis of the condyle. T1 images revealed a discrete low-signal area surrounded by an area of intermediate intensity signal. A serpiginous low-level signal line was present at the margin of the lesion. T2 images revealed high-level signal intensity at the lesional edge, accompanied by bone marrow edema. (Fig. 4).

Retrospectively, we carefully compared the knee MRI of 2013 with that of 2015, and which surprised us was that osteonecrosis of subchondral bone of the medial femoral condyle seems had appeared in 2013, because T1 images exhibited a tiny low-signal region in the bone where T2 images showed a slight high intensity. (Fig. 5). Thus, we confirmed that SONK had onset before arthroscopic surgery and diagnosis of the SONK could be established based on the history, physical examination and imaging studies.

Moreover, the focal necrotic volume was $0.894 \mathrm{~cm} 3$ now according to the measurement results and the patient could barely walk. Hence, we performed unicompartmental arthroplasty, which completely relieved the pain and restored walking ability of the patient. (Fig. 6).

\section{Discussion And Conclusions}

Spontaneous osteonecrosis of the knee is a disorder of uncertain etiology, classically described as a focal lesion occurring in the medial femoral condyle of a patient in the fifth or sixth decade of life, with females affected 3-5-fold more commonly than males[2]. Studies reported that the prevalence of earlystage SONK in patients $>65$ years of age with medial knee pain but without a trauma history was $9.4 \%[3]$. Patients typically present with sudden-onset severe pain in the medial aspect of the knee just proximal to the joint line. Although traumatic etiologies have been reported, only a minority of patients recall specific injuries that precipitated symptoms[4]. In the acute phase of disease, patients often report pain on weight bearing and an increase in pain severity at night. Depending on the stage and size of the lesion, the acute pain either gradually resolves or becomes chronically debilitating.

A plain X-ray may reveal a radiolucent lesion with a surrounding sclerotic halo and subtle flattening of the involved femoral condyle. In advanced cases exhibiting significant subchondral collapse, secondary degenerative changes may be evident. These include loss of joint space, sclerosis of the medial tibial plateau, and osteophyte formation. On MRI, T1 imaging reveals a discrete low-signal area often 
surrounded by an area of intermediate signal intensity. A serpiginous low-signal line is often present at the margin of the lesion, delineating the necrotic area from an adjacent region of bone marrow edema. T2 images are typically of high signal intensity at the lesional edge, in the region of the edema[5]. When patients yield normal radiographs after sudden-onset severe knee pain, MRI should be performed no earlier than 6 weeks after symptom onset to rule out pre-existing early-stage SONK[3]. A SONK diagnosis may avoid unnecessary arthroscopy.

The presence of a medial meniscal tear has been proposed as a potential etiology of SONK[6]. Such tears were evident in $50-78 \%$ of SONK patients in the recent series of Robertson[7]. Tears in the meniscal root were evident in 24 of 30 patients (80\%). The cited authors suggested that, in elderly patients with osteoporotic bones, discontinuity of the medial meniscus was associated with inappropriate hoop stress distribution in the medial compartment, thus increasing the load on the femoral condyle, potentially predisposing to the development of subchondral insufficiency fractures. The plain X-ray yields useful prognostic data, particularly with regard to the lesional size. In many early studies, the lesional area within the condyle successfully predicted progression to severe degenerative arthritis.

In addition to SONK, there is also secondary osteonecrosis. Distinguishing from SONK, secondary osteonecrosis is associated with predisposing factors including corticosteroid use, rheumatoid arthritis, alcohol consumption, sickle-cell disease, systemic lupus erythematosus, Caisson's disease, and Gaucher's disease $[8,9]$. Bilateral joint involvement is evident in $30-80 \%$ of patients and the lateral femoral condyle is affected in $60 \%[10]$. Multifocal osteonecrosis with simultaneous involvement of the knees, hips, and shoulders has also been reported[8, 9]. Lesional specimens are large and wedge-shaped. MRI reveals a demarcation rim at the border between necrotic and viable bone. Furthermore, postarthroscopic osteonecrosis belongs to a type of secondary osteonecrosis. Some postarthroscopic may develope after arthroscopic meniscectomy, shaver-assisted chondroplasty, anterior cruciate ligament reconstruction, and laser-or radiofrequency-assisted debridement. The condition usually affects the epiphyseal region of a single condyle in the operated knee and develops at a mean of approximately 24 weeks after surgery ( Range: 4-92 weeks)[11]. It has been proposed that laser- or radiofrequency-assisted arthroscopic surgery may trigger osteonecrosis via direct thermal injury or photoacoustic shock[12]. Patients usually present with acute-onset or worsening knee pain and a recent history of therapeutic arthroscopic surgery. As most have undergone such surgery to treat knee pain, the pain associated with secondary osteonecrosis may be mistaken as treatment failure or recurrence[4].

Conservative treatment is recommended in the early stage of disease; this includes anti-inflammatory drugs, lateral-wedge insoles, and protected weight bearing[13]. Typically, restrictions on weight bearing are maintained for 4-8 weeks. As symptoms improve, a resumption of normal daily activities is allowed, and physical therapy (quadriceps and hamstring strengthening) is initiated[14]. In patients with stage 2 disease (thus, with relatively intact articular cartilage), drilling may be helpful, stimulating mesenchymal stem cell (MSC) proliferation and subsequent reductions in interosseous pressure[15]. Some investigators have used retrograde arthroscopic drilling to this end, stimulating lesional revascularization[16]. However, such drilling may damage the articular surface. Also, the difficulty associated with accurate localization 
of the lesional focus in the pre-collapse stage renders antegrade drilling or core decompression more attractive. As the primary pathology is intraosseous, arthroscopic debridement is unlikely to change the course of disease.

Patients with advanced-stage disease generally require surgery with placement of osteochondral grafts, high tibial osteotomy (HTO) with or without autogenic bone grafting, unicompartmental knee arthroplasty (UKA), or total knee arthroplasty (TKA); and enjoy good clinical outcomes. Appropriately selected patients may be managed via HTO to preserve the joint[17]. Although this option is typically reserved for younger, active patients, HTO relieves the affected femoral condyle by shifting the weight-bearing axis laterally. In a study on 10 patients managed either via HTO[10] or non-operatively[8]. Study have found that HTO was associated with greater improvements in lesional appearance on follow-up MRI (83\% vs. $25 \%$ ) and a higher frequency of symptom improvement (100\% vs. $50 \%)[18]$.

For patients in whom joint-preserving treatments fail to provide symptomatic improvement and in those with large or advanced lesions, arthroplasty is the treatment of choice. Depending on individual patient factors, lesional characteristics, and the condition of the remainder of the joint, either unicompartmental arthroplasty or standard TKA may be indicated. Unicompartmental arthroplasty is effective in those in whom disease is isolated to a single femoral condyle or the tibial plateau, preserving both bone stock and functional cruciate ligaments[19, 20, 21].

In conclusion, we report a case of an early-stage SONK misdiagnosed as meniscus injury. SONK is a progressive disorder and the treatment is stage-specific. This case remind us that orthopedic surgeons must know the patient population of the risk, the classic presentation and the radiological characteristics. A high index of suspicion and distinction are required in the diagnosis of knee pain. Accompanying meniscal tear may be a characteristic of SONK which would create a disturbance in the diagnosis. Of note, early diagnosis and suitable treatment could improve the clinical outcome.

\section{Abbreviations}

MSC: Mesenchymal stem cell; SONK: Spontaneous osteonecrosis of the knee; MRI: Magnetic resonance imaging; HTO: High tibial osteotomy; UKA: Unicompartmental knee arthroplasty; TKA: Total knee arthroplasty.

\section{Declarations}

\section{Ethics approval and consent to participate}

Not applicable

\section{Consent for publication}


The authors have obtained the patient's written informed consent for print and electronic publication of this case report

\section{Availability of data and materials}

This is a case report of a single patient, to protect privacy and respect confidentiality; none of the raw data has been made available in any public repository. The original data are retained as per normal procedure within the medical records of our institution. The datasets used and/or analysed during the current study are available from the corresponding author on reasonable request.

\section{Authors' contributions}

WMY and XWC contributed to the conception and design of the study. WMY and CQZ collected and analyzed date, drafted and critically revised the manuscript for important intellectual content. XWC supervised the manuscript drafting and data collection. CQZ analyzed the radiological features. WMY and CQZ are responsible for the oversight of the report and editing the manuscript. All authors read and finally approved the manuscript.

\section{Competing Interests}

The authors declare that they have no competing interests.

\section{Funding}

None

\section{Acknowledgements}

Not applicable

\section{References}

1. Ahlback S, Bauer GC, Bohne WH. Spontaneous osteonecrosis of the knee. Arthritis Rheum. 1968; 11(6):705-33.

2. Kattapuram TM, Kattapuram SV. Spontaneous osteonecrosis of the knee. EUR J RADIOL. 2008; 67(1):42-8.

3. Pape D, Seil R, Fritsch E, Rupp S, Kohn D. Prevalence of spontaneous osteonecrosis of the medial femoral condyle in elderly patients. Knee Surg Sports Traumatol Arthrosc. 2002; 10(4):233-40.

4. Zywiel MG, McGrath MS, Seyler TM, Marker DR, Bonutti PM, Mont MA. Osteonecrosis of the knee: a review of three disorders. Orthop Clin North Am. 2009; 40(2):193-211.

5. Sung JH, Ha JK, Lee DW, Seo WY, Kim JG. Meniscal extrusion and spontaneous osteonecrosis with root tear of medial meniscus: comparison with horizontal tear. ARTHROSCOPY. 2013; 29(4):726-32. 
6. Muscolo DL, Costa-Paz M, Ayerza M, Makino A. Medial meniscal tears and spontaneous osteonecrosis of the knee. ARTHROSCOPY. 2006; 22(4):457-60.

7. Robertson DD, Armfield DR, Towers JD, Irrgang JJ, Maloney WJ, Harner CD. Meniscal root injury and spontaneous osteonecrosis of the knee: an observation. J Bone Joint Surg Br. 2009; 91(2):190-5.

8. Mont MA, Baumgarten KM, Rifai A, Bluemke DA, Jones LC, Hungerford DS. Atraumatic osteonecrosis of the knee. J BONE JOINT SURG AM. 2000; 82(9):1279-90.

9. Narvaez J, Narvaez JA, Rodriguez-Moreno J, Roig-Escofet D. Osteonecrosis of the knee: differences among idiopathic and secondary types. Rheumatology (Oxford). 2000; 39(9):982-9.

10. Yamamoto T, Bullough PG. Spontaneous osteonecrosis of the knee: the result of subchondral insufficiency fracture. J BONE JOINT SURG AM. 2000; 82(6):858-66.

11. Bonutti PM, Seyler TM, Delanois RE, McMahon M, McCarthy JC, Mont MA. Osteonecrosis of the knee after laser or radiofrequency-assisted arthroscopy: treatment with minimally invasive knee arthroplasty. J BONE JOINT SURG AM. 2006; 88 Suppl 3:69-75.

12. Lee EW, Paulos LE, Warren RF. Complications of thermal energy in knee surgery-Part II. Clin Sports Med. 2002; 21(4):753-63.

13. Breer S, Oheim R, Krause M, Marshall RP, Amling M, Barvencik F. Spontaneous osteonecrosis of the knee (SONK). Knee Surg Sports Traumatol Arthrosc. 2013; 21(2):340-5.

14. Strauss EJ, Kang R, Bush-Joseph C, Bach BJ. The diagnosis and management of spontaneous and post-arthroscopy osteonecrosis of the knee. Bull NYU Hosp Jt Dis. 2011; 69(4):320-30.

15. Akgun I, Kesmezacar H, Ogut T, Kebudi A, Kanberoglu K. Arthroscopic microfracture treatment for osteonecrosis of the knee. ARTHROSCOPY. 2005; 21(7):834-43.

16. DeFalco RA, Ricci AR, Balduini FC. Osteonecrosis of the knee after arthroscopic meniscectomy and chondroplasty: a case report and literature review. Am J Sports Med. 2003; 31(6):1013-6.

17. Duany NG, Zywiel MG, McGrath MS, Siddiqui JA, Jones LC, Bonutti PM, Mont MA. Joint-preserving surgical treatment of spontaneous osteonecrosis of the knee. Arch Orthop Trauma Surg. 2010; 130(1):11-6.

18. Marti CB, Rodriguez M, Zanetti M, Romero J. Spontaneous osteonecrosis of the medial compartment of the knee: a MRI follow-up after conservative and operative treatment, preliminary results. Knee Surg Sports Traumatol Arthrosc. 2000; 8(2):83-8.

19. Bruni D, lacono F, Raspugli G, Zaffagnini S, Marcacci M. Is unicompartmental arthroplasty an acceptable option for spontaneous osteonecrosis of the knee? Clin Orthop Relat Res. 2012; 470(5):1442-51.

20. Heyse TJ, Khefacha A, Fuchs-Winkelmann S, Cartier P. UKA after spontaneous osteonecrosis of the knee: a retrospective analysis. Arch Orthop Trauma Surg. 2011; 131(5):613-7.

21. Yang WM, Zhao CQ, Lu ZY, Yang WY,Lin DK, Cao XW. Clinical Characteristics and Treatment of Spontaneous Osteonecrosis of Medial Tibial Plateau: A Retrospective Case Study. Chin Med J 2018;131:2544-50. 
Figures
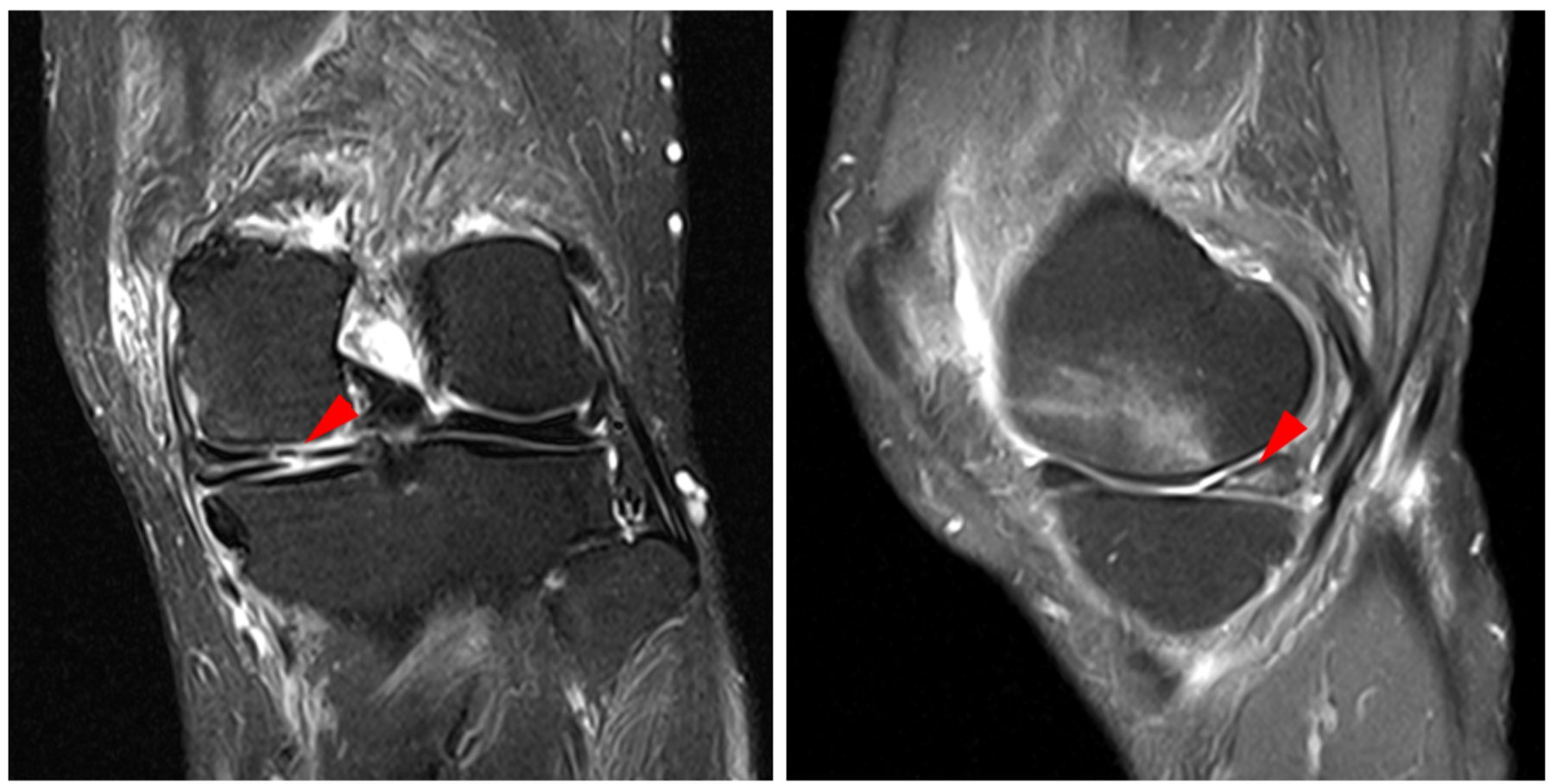

Figure 1

MRI of left knee revealed a medial meniscal root tear (level III) in december 2013, as indicated by red arrows. 

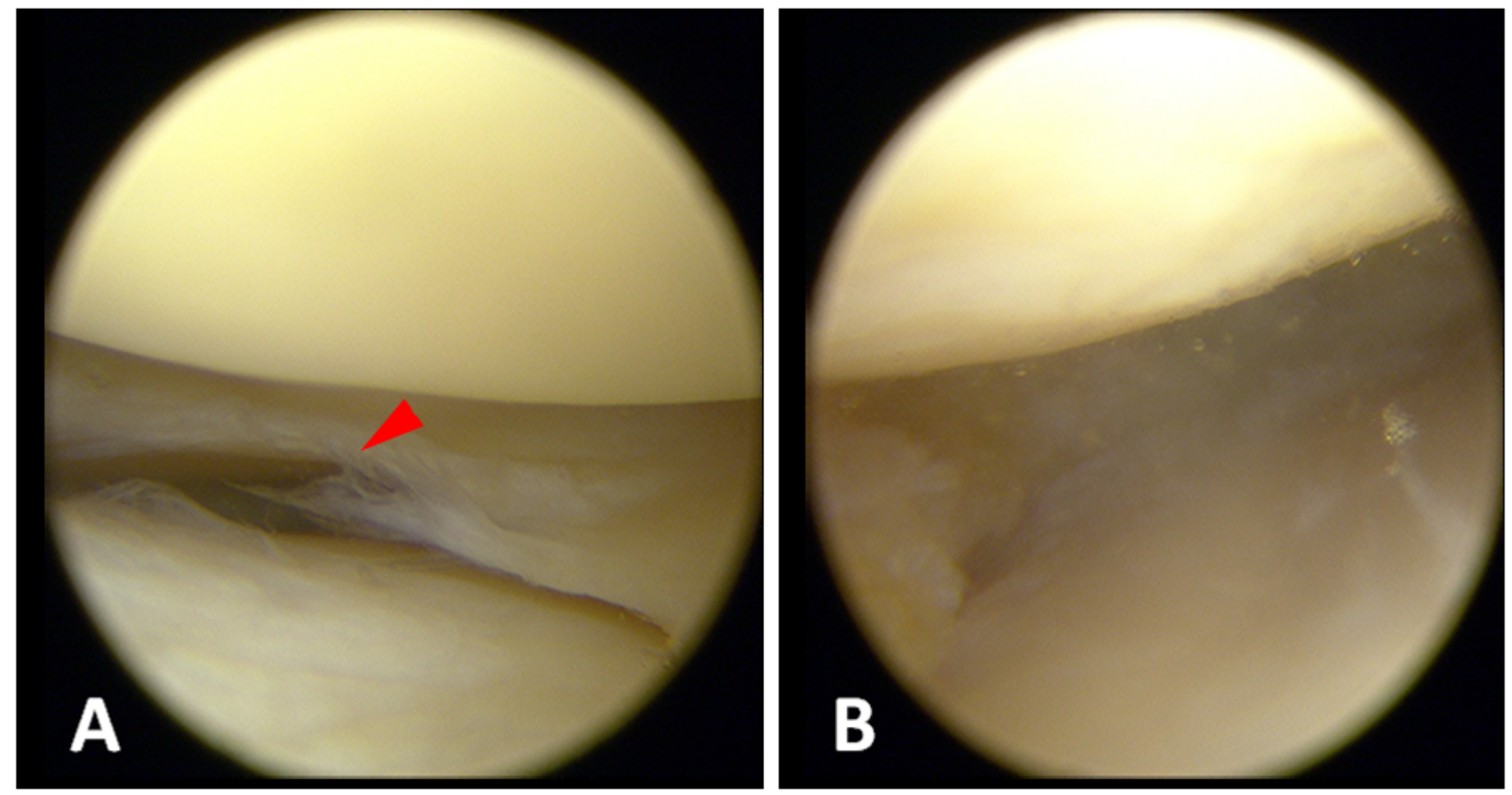

Figure 2

Arthroscopic surgery was performed. Intraoperative inspection revealed that the medial meniscal root exhibited a radial tear (red arrows). 

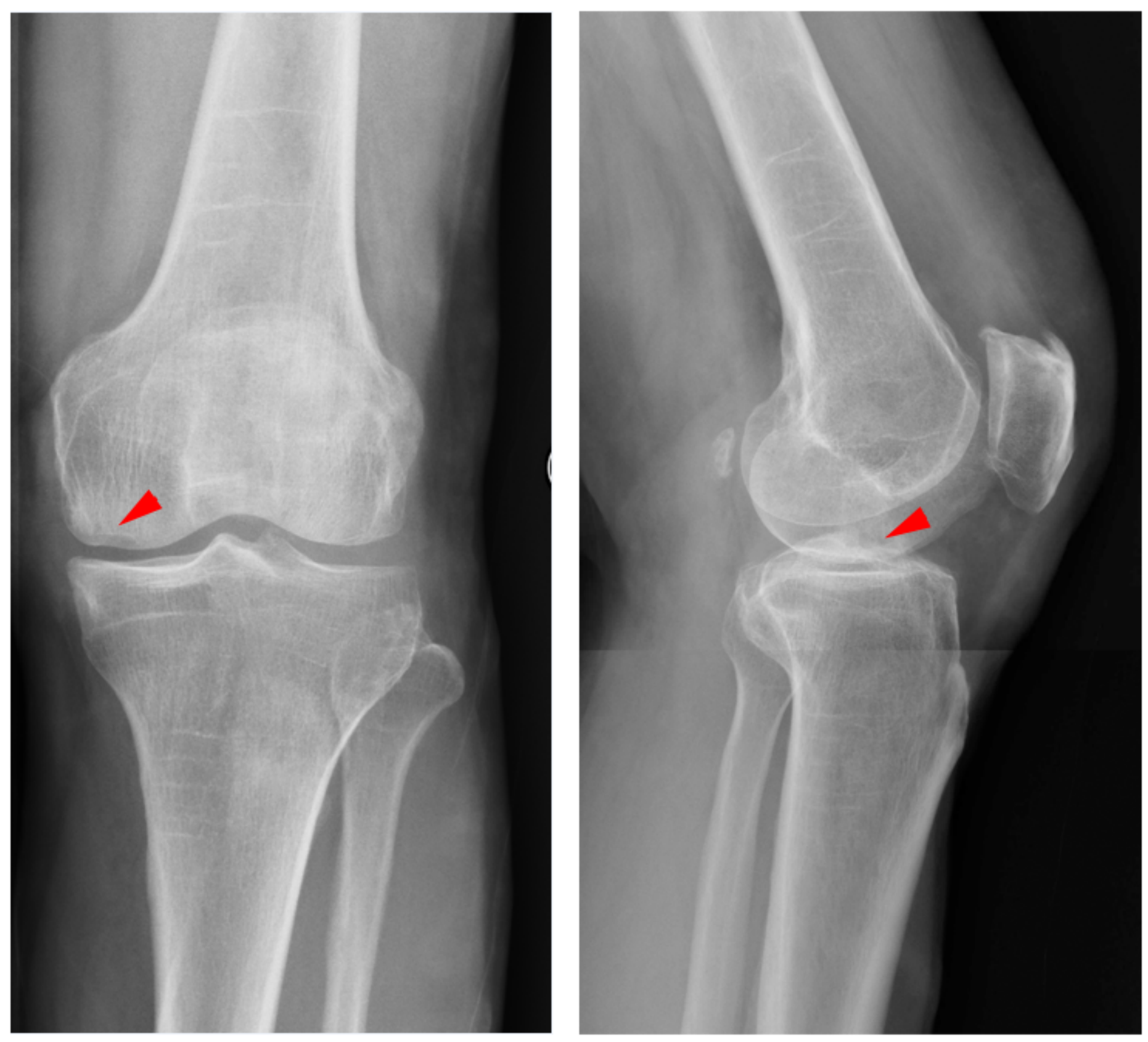

Figure 3

$X$ ray in May 2015 revealed a radiolucent lesion with a surrounding sclerotic halo and subtle flattening of the involved femoral condyle. As indicated by red arrows. 

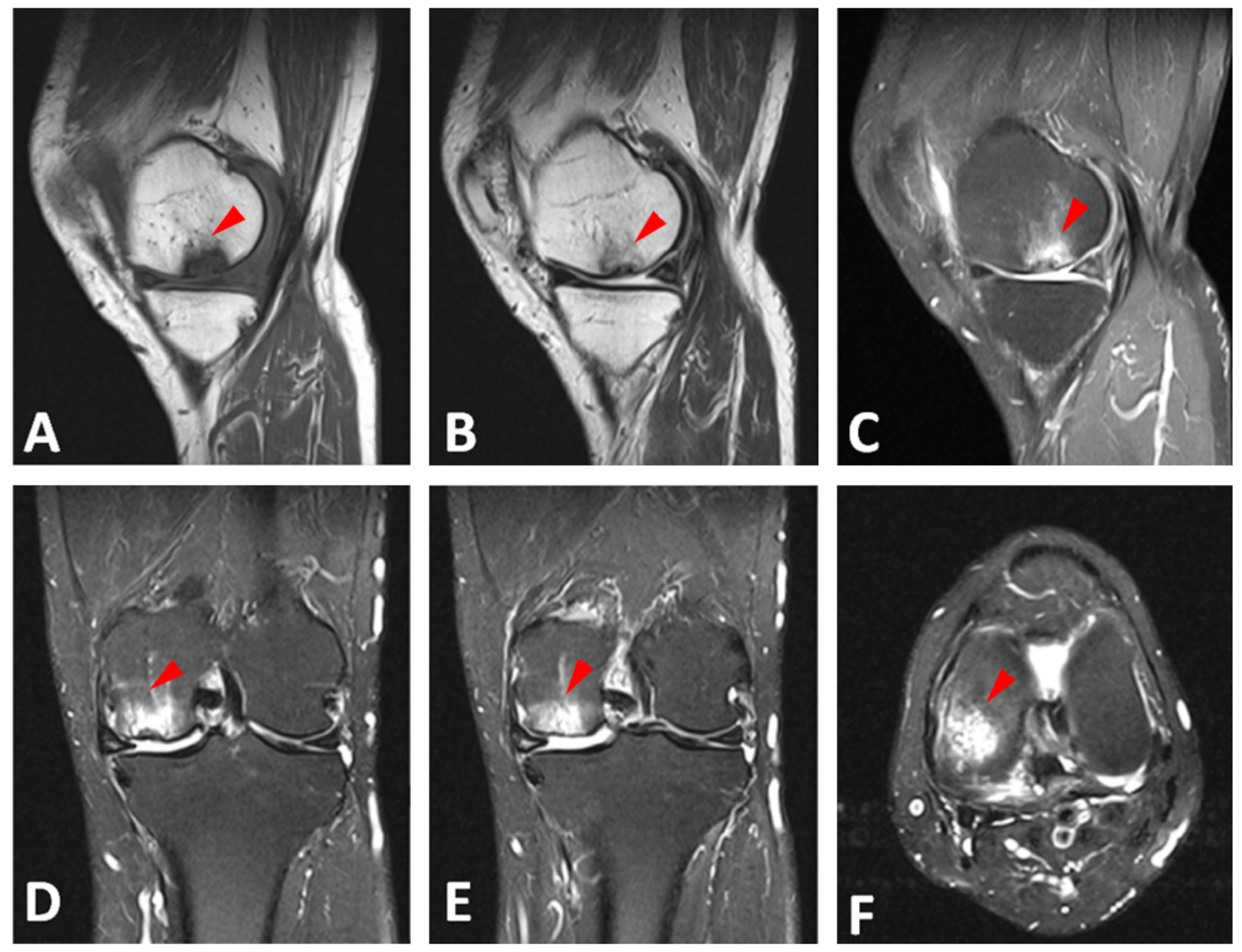

Figure 4

MRI in May 2015. The T1 images showed a discrete low-signal area surrounded by an area of intermediate signal intensity. A serpiginous low-level signal line was evident at the margin of the lesion (A and $B$, red arrows). T2 images exhibited high-level signal intensity at the lesional edge, associated with bone marrow edema (C-F, red arrows). 

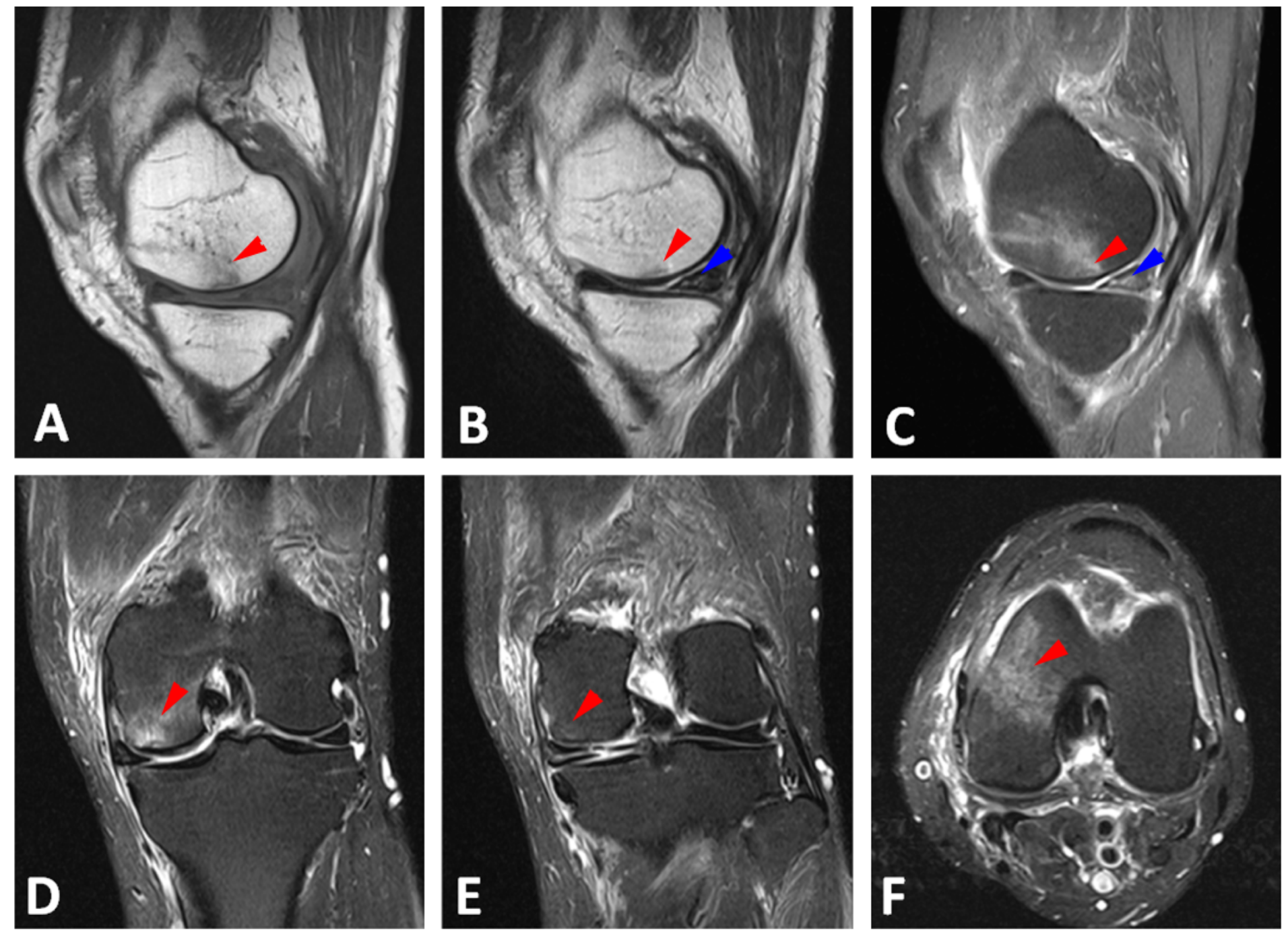

Figure 5

MRI in 2013 revealed a medial meniscal root tear (level III), (B and C, blue arrows). The T1 images exhibited a tiny low-signal region in subchondral bone of the medial femoral condyle ( $A$ and $B$, red arrows). The T2 images showed slight elevated signal intensity in femoral condyle (C-F, red arrows). 


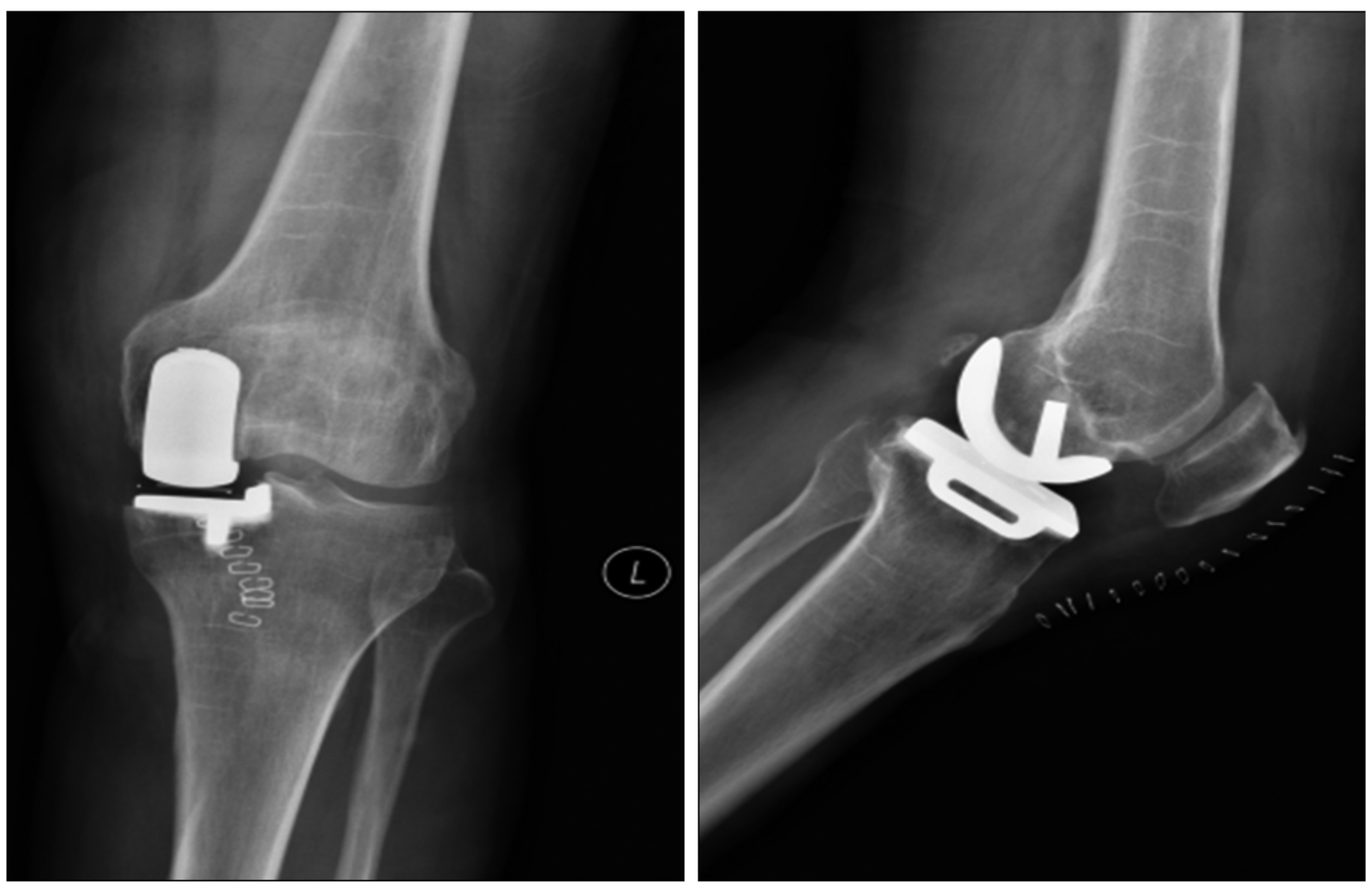

Figure 6

The $\mathrm{X}$ ray taken after unicompartmental arthroplasty. 